PROCEEDINGS OF THE

AMERICAN MATHEMATICAL SOCIETY

Volume 125, Number 12, December 1997, Pages 3457-3463

S 0002-9939(97)04332-3

\title{
COMPUTING CONGRUENCE LATTICES OF FINITE LATTICES
}

\author{
RALPH FREESE
}

(Communicated by Lance W. Small)

\begin{abstract}
An inequality between the number of coverings in the ordered set $\mathrm{J}$ (Con $\mathbf{L}$ ) of join irreducible congruences on a lattice $\mathbf{L}$ and the size of $\mathbf{L}$ is given. Using this inequality it is shown that this ordered set can be computed in time $O\left(n^{2} \log _{2} n\right)$, where $n=|L|$.
\end{abstract}

This paper is motivated by the problem of efficiently calculating and representing the congruence lattice Con $\mathbf{L}$ of a finite lattice $\mathbf{L}$. Of course Con $\mathbf{L}$ can be exponential in the size of $\mathbf{L}$; for example, when $\mathbf{L}$ is a chain of length $n$, Con $\mathbf{L}$ has $2^{n}$ elements. However, since $\mathbf{C o n} \mathbf{L}$ is a distributive lattice, it can be recovered easily from the ordered set of its join irreducible elements $\mathrm{J}(\mathbf{C o n} \mathbf{L})$. Indeed any finite distributive lattice $\mathbf{D}$ is isomorphic to the lattice of order ideals of $J(\mathbf{D})$ and this lattice is in turn isomorphic to the lattice of all antichains of $\mathrm{J}(\mathbf{D})$, where the antichains are ordered by $A \ll B$, i.e., for each $a \in A$ there is a $b \in B$ with $a \leq b$. If $\mathbf{P}$ is an ordered set of size $n$ which has $N$ order ideals, then there are straightforward algorithms to find the order ideals of $\mathbf{P}$ which run in time $O(n N)$; see, for example, [5]. In [10] Medina and Nourine give an algorithm which runs in time $O(d N)$, where $d$ is the maximum number of covers of any element of $\mathbf{P}$. Thus we will concentrate on the problem of efficiently finding $\mathrm{J}(\operatorname{Con} \mathbf{L})$.

\section{Preliminaries}

Throughout this paper $\mathbf{L}$ denotes a finite lattice. $\mathrm{J}(\mathbf{L})$ denotes the set of nonzero join irreducible elements and $\mathrm{M}(\mathbf{L})$ the set of nonunit meet irreducible elements. These sets are ordered by the induced order from $\mathbf{L}$. If $a \in \mathrm{J}(\mathbf{L})$, then it has a unique lower cover in $\mathbf{L}$ which we denote by $a_{*}$, and similarly if $q \in \mathrm{M}(\mathbf{L})$, then $q^{*}$ is the unique upper cover of $q$. The cover relation is denote by $a \prec b$; $\mathrm{Cg}(x, y)$ is the smallest congruence identifying $x$ and $y$. Throughout the paper we let

$$
n=|L| \quad m=\mid \mathrm{J}(\text { Con } \mathbf{L}) \mid \text {. }
$$

For an ordered set $\mathbf{P}$ we define

$$
\begin{array}{ll}
E_{\leq}=\{\langle a, b\rangle: a \leq b\}, & e_{\leq}=\left|E_{\leq}\right|, \\
E_{\prec}=\{\langle a, b\rangle: a \prec b\}, & e_{\prec}=\left|E_{\prec}\right| .
\end{array}
$$

Received by the editors June 11, 1996.

1991 Mathematics Subject Classification. Primary 06B10, 06B05, 06B15.

Key words and phrases. Congruence lattice, algorithm.

This research was partially supported by NSF grant no. DMS-9500752. 
For clarity we sometimes write $E_{\leq}(\mathbf{P})$, etc. Of course $\mathbf{P}$ is determined from its underlying set $P$ and any 'edge' set $E$ with

$$
E_{\prec} \subseteq E \subseteq E_{\leq} .
$$

The transitive, reflexive closure of any such $E$ is $E_{\leq}$, and $E_{\prec}$ is the smallest set whose transitive, reflexive closure is $E_{\leq} . E_{\prec}$ is alternatively known as the covering relation, the transitive reduct, and the Hasse diagram of $\mathbf{P}$. We are most interested in the case $\mathbf{P}=\mathrm{J}($ Con $\mathbf{L})$ and in this paper $E_{\leq}=E_{\leq}(\mathrm{J}($ Con $\mathbf{L}))$, etc.

Chapter XI of [5] contains a discussion of algorithms for lattice theory including algorithms for calculating the congruence lattice of a finite lattice. It shows there is an algorithm which computes the set $\mathrm{J}(\mathbf{C o n} \mathbf{L})$ and an edge set $E$ satisfying (2) in time $O\left(n^{2}\right)$. Of course $\mathbf{L}$ is simple exactly when $\mathrm{J}(\mathbf{C o n} \mathbf{L})$ has only one element, and it is subdirectly irreducible if it has only one minimal element. Since the minimal elements can be found quickly from any $E$ satisfying (2), this gives $O\left(n^{2}\right)$ algorithms for testing the simplicity and subdirect irreducibility of $\mathbf{L}$. However, it does not give the full transitive, reflexive closure, $E_{\leq}$, which is needed for other purposes.

By Theorem 11.2 of [5] we can calculate $E_{\leq}$from any $E$ satisfying (2) (not just $\left.E_{\prec}\right)$ in time $O\left(m^{2}+m e_{\prec}+e_{\leq}\right)$. It follows from part 3 of Lemma 1 below that $m \leq n$ and thus $E_{<}$can be found in time $O\left(n^{3}\right)$. The main purpose of this paper is to improve this bound. We will show that $E_{\leq}$can be found in time $O\left(n^{2} \log _{2} n\right)$.

In order to do this we explore the connection between $\mathbf{L}$ and $\mathbf{J}(\mathbf{C o n} \mathbf{L})$ more carefully. Namely, given a number $e_{\prec}$, we would like to know how small $\mathbf{L}$ can be with $e_{\prec}=e_{\prec}(\mathrm{J}(\mathbf{C o n} \mathbf{L}))$. In [8] and [9] Grätzer, Lakser, Rival, Schmidt, and Zaguia investigate the relation between $m$ and $n$. As mentioned above, $m \leq n$. In [8] it is shown that for any ordered set $\mathbf{P}$ of size $m$ there is a lattice $\mathbf{L}$ of size $O\left(m^{2}\right)$ with $\mathrm{J}($ Con $\mathbf{L}) \cong \mathbf{P}$. In [9] examples are given showing that no smaller power of $m$ will work.

For our purposes we need a universal lower bound on the size of $\mathbf{L}$ in terms of $e_{\prec}$. What we show is that given an ordered set $\mathbf{P}$ with $e_{\prec}$ covers, any $n$-element lattice $\mathbf{L}$ with $\mathrm{J}(\mathbf{C o n} \mathbf{L}) \cong \mathbf{P}$ has

$$
e_{\prec} \leq 2 n \log _{2} n
$$

We give examples showing that in a certain sense this is the best possible and we show how to derive the result of [9] as a corollary.

\section{The INEQUALITY}

We begin with some lemmas. The first is elementary and well known; the second is new; the third is from [9].

Lemma 1. Let $\mathbf{L}$ be a finite lattice.

1. If $a \prec b$ in $\mathbf{L}$, then $\operatorname{Cg}(a, b)$ is join irreducible.

2. If $\theta \in \mathrm{J}(\mathbf{C o n} \mathbf{L})$, then there exist $a \in \mathrm{J}(\mathbf{L})$ and $q \in \mathrm{M}(\mathbf{L})$ with $\theta=$ $\mathrm{Cg}\left(a, a_{*}\right)=\mathrm{Cg}\left(q, q^{*}\right)$.

3. The function $a \mapsto \operatorname{Cg}\left(a, a_{*}\right)$ maps $\mathrm{J}(\mathbf{L})$ onto $\mathrm{J}(\mathbf{C o n} \mathbf{L})$.

Proof. The first statement follows easily from Dilworth's characterization of lattice congruences, Theorem 10.2 of [1], and does not require that $\mathbf{L}$ be finite. Again by Dilworth's theorem any (completely) join irreducible congruence $\theta$ has the form $\mathrm{Cg}(x, y)$ for some $x>y$. Since $\mathbf{L}$ is finite, there is a finite maximal chain from $x$ 
down to $y$, and since $\theta$ is join irreducible, one of the links of the chain must generate $\theta$. So we may assume $x \succ y$ and if we choose $q$ to be maximal above $y$ but not $x$, then $q$ is meet irreducible and $\theta=\operatorname{Cg}\left(q, q^{*}\right)$, as desired. The third statement follows from the second.

Lemma 2. Suppose $\theta \prec \phi$ in $\mathrm{J}(\mathbf{C o n} \mathbf{L})$. Then one of the following holds.

1. There is an $a \in \mathrm{J}(\mathbf{L})$ and $x \in L$ with $a \succ a_{*}>x$ such that $\operatorname{Cg}\left(a, a_{*}\right)=\theta$ and $\mathrm{Cg}\left(a_{*}, x\right)=\phi$.

2. There is a $q \in \mathrm{M}(\mathbf{L})$ and $x \in L$ with $q \prec q^{*}<x$ such that $\mathrm{Cg}\left(q, q^{*}\right)=\theta$ and $\operatorname{Cg}\left(q^{*}, x\right)=\phi$.

Proof. For $a, b \in \mathrm{J}(\mathbf{L})$ and $q \in \mathrm{M}(\mathbf{L})$ we define two relations by

$$
\begin{aligned}
& a \nearrow q \text { if and only if } \quad a \leq q^{*} \text { and } a \not \leq q, \\
& q \searrow b \text { if and only if } \quad q \geq b_{*} \text { and } q \nsupseteq b .
\end{aligned}
$$

Note that $a \nearrow q$ if and only if $a \vee q=q^{*}$, and $q \searrow b$ if and only if $q \wedge b=b_{*}$. Define $\mathbf{G}(\mathbf{L})=\langle V, \rightarrow\rangle$ to be the directed graph whose vertex set $V$ is the disjoint union of (copies of) $\mathrm{J}(\mathbf{L})$ and $\mathrm{M}(\mathbf{L})$ and whose relation $\rightarrow$ is the union of $\nearrow$ and $\searrow$. Of course this graph determines a quasiorder. Let $\equiv$ be the equivalence relation for this quasiorder: $x \equiv y$ if and only if $x \rightarrow^{*} y$ and $y \rightarrow^{*} x$, where $\rightarrow^{*}$ represents a sequence, possibly of length 0 , of edges. We let $\mathbf{G}(\mathbf{L}) / \equiv$ denote the induced ordered set on the equivalence classes. By Lemma 11.11 of [5] $\mathbf{G}(\mathbf{L}) / \equiv$ is isomorphic $\mathrm{J}(\mathbf{C o n} \mathbf{L})$.

Since $\phi \succ \theta$, there must be an edge of $\mathbf{G}(\mathbf{L})$ going from an element in the $\equiv$-class corresponding to $\theta$ to an element in the class corresponding to $\phi$. Suppose this edge has the form (3). This situation is diagrammed in Figure 1.

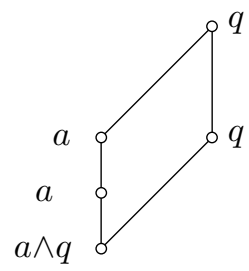

FiguRE 1

If $a \wedge q=a_{*}$, then we would have $\theta=\operatorname{Cg}\left(a, a_{*}\right)=\operatorname{Cg}\left(q, q^{*}\right)=\phi$, a contradiction. Thus $a \wedge q<a_{*}$ and the first statement of the lemma holds with $x=a \wedge q$. Of course if the the edge has the form (4), then the second statement of the lemma will hold.

Lemma 3. Let $\mathbf{L}$ be a lattice, let $A \subseteq L$, and let $b \in L$ be a lower bound of $A$ in $\mathbf{L}$. Assume that $\mathrm{Cg}(b, a)$ is join irreducible and that $\mathrm{Cg}(b, a)$ and $\mathrm{Cg}\left(b, a^{\prime}\right)$ are incomparable for each $a \neq a^{\prime}$ in $A$. Then $A$ is join irredundant.

Proof. This is proved in [9].

Theorem 4. Let $\mathbf{L}$ be a finite lattice. Then

$$
e_{\prec}(\mathrm{J}(\mathbf{C o n} \mathbf{L})) \leq(|\mathrm{J}(\mathbf{L})|+|\mathrm{M}(\mathbf{L})|) \log _{2}|L| \leq 2|L| \log _{2}|L| .
$$


Proof. Let $r=|\mathrm{J}(\mathbf{L})|+|\mathrm{M}(\mathbf{L})|$ and $e_{\prec}=e_{\prec}(\mathrm{J}($ Con $\mathbf{L}))$ and $t=\left\lceil e_{\prec} / r\right\rceil$. By Lemma 2, associated with each cover in $\mathrm{J}($ Con $\mathbf{L})$ is an element of $L$ which is either join or meet irreducible. Hence there is either a join irreducible element $a$ satisfying the first possible conclusion of that lemma or a meet irreducible one satisfying the second. Thus by duality we may assume that there is a meet irreducible element $q$ and elements $x_{1}, \ldots, x_{t}$ with $q^{*}<x_{i}$ such that $\phi_{i}=\operatorname{Cg}\left(x_{i}, q^{*}\right)$ covers $\theta=\operatorname{Cg}\left(q, q^{*}\right)$. Thus $\phi_{1}, \ldots, \phi_{t}$ form an antichain. So by Lemma $3 x_{1}, \ldots, x_{t}$ are join irredundant. Thus $2^{t} \leq|L|$ and so $e_{\prec} / r \leq \log _{2}|L|$, and the theorem follows.

Corollary 5. If $\mathbf{L}$ is a lattice with $n$ elements, then one can determine the ordered set $\mathrm{J}(\mathbf{C o n} \mathbf{L})$ and its order relation in time $O\left(n^{2} \log _{2} n\right)$.

Proof. As we noted before we can find $\mathrm{J}($ Con $\mathbf{L})$ and its order relation in time $O\left(m^{2}+m e_{\prec}+e_{\leq}\right)$. Since $e_{\prec} \leq 2 n \log _{2} n$ and $m \leq n$, the result follows.

Since the function $x / \log _{2} x$ is increasing for $x \geq e$ (Euler's constant), we get the following corollary to Theorem 4 .

Corollary 6. Let $\mathbf{L}$ be a finite lattice and let $e_{\prec}=e_{\prec}(\mathrm{J}(\mathbf{C o n} \mathbf{L}))$. If $e_{\prec} \geq 3$, then

$$
|L| \geq \frac{e_{\prec}}{2 \log _{2} e_{\prec}} .
$$

\section{EXAMPLES}

In this section we give some examples showing that the inequality of Theorem 4 cannot be improved. We also show that this theorem easily gives the result of [9].

Example 7. Let $r_{1}, r_{2}$, and $s$ be positive integers and let $\mathbf{K}_{i}=\mathbf{3} \times \mathbf{2}^{r_{i}-1}$ for $i=1$, 2. Of course $\mathbf{K}_{1}$ has a filter isomorphic to the three element chain $\mathbf{3}$ and $\mathbf{K}_{2}$ has an ideal isomorphic to $\mathbf{3}$. Using the gluing construction of Dilworth and Hall [3, 4], we can identify these two copies of $\mathbf{3}$ and use the natural order to obtain a lattice $\mathbf{K}$. Let $x$ denote the middle element of this three element chain in $\mathbf{K}$. Replace $x$ with $x_{0}<x_{1}<\cdots<x_{s}$ and order the resulting set by saying $z<x_{i}<y$ whenever $z<x<y$ in $\mathbf{K}$. Let $\mathbf{L}$ denote the resulting lattice. Note $\mathbf{L}$ is obtained from $\mathbf{K}$ by $s$ applications of Alan Day's doubling construction [2]. The diagram of $\mathbf{L}$ is given in Figure 2 when $r_{1}=r_{2}=3$ and $s=3$.

Con $\mathbf{L}$ is the lattice obtained by placing the Boolean algebra $\mathbf{2}^{r}$ on top of the the Boolean algebra $\mathbf{2}^{s}$, where $r=r_{1}+r_{2} . \mathrm{J}(\mathbf{C o n} \mathbf{L})$ is the complete bipartite ordered set with $r$ top elements and $s$ bottom elements. So

$$
\begin{gathered}
|L|=s+3 \cdot 2^{r_{1}-1}+3 \cdot 2^{r_{2}-1}-3 \\
e_{\prec}(\mathrm{J}(\mathbf{C o n} \mathbf{L}))=r s \\
|\mathrm{~J}(\mathbf{L})|+|\mathrm{M}(\mathbf{L})|=2 r+2 s .
\end{gathered}
$$

Let $e_{\prec}$ be an integer such that both $\log _{2} e_{\prec}$ and $e_{\prec} / \log e_{\prec}$ are integers and let $r_{1}=r_{2}=\log _{2} e_{\prec}$ (and so $r=2 \log _{2} e_{\prec}$ ) and $s=e_{\prec} / r$. (This means $e_{\prec}$ has the 


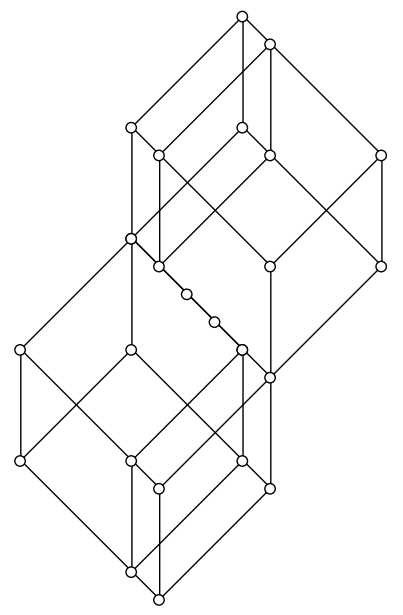

Figure 2. L when $r_{1}=r_{2}=3$ and $s=3$.

form $2^{2^{k}}$.) Then we have by Theorem 4

$$
\begin{aligned}
1 & \leq \frac{\left(2 e_{\prec} / 2 \log _{2} e_{\prec}+4 \log _{2} e_{\prec}\right) \log _{2}\left(6 \cdot 2^{\log _{2} e_{\prec}-1}-3+e_{\prec} / 2 \log _{2} e_{\prec}\right)}{e_{\prec}} \\
& \leq \frac{\left(2 e_{\prec} / 2 \log _{2} e_{\prec}+4 \log _{2} e_{\prec}\right) \log _{2}\left(3 e_{\prec}+e_{\prec} / 2 \log _{2} e_{\prec}\right)}{e_{\prec}} .
\end{aligned}
$$

But the latter expression tends to 1 as $e_{\prec}$ tends to $\infty$. It follows that Theorem 4 cannot be improved to state

$$
e_{\prec}(\mathrm{J}(\mathbf{C o n} \mathbf{L})) \leq c(|\mathrm{~J}(\mathbf{L})|+|\mathrm{M}(\mathbf{L})|) \log _{2}|L|
$$

for any $c<1$.

If we take $r_{1}=r_{2}=\frac{1}{2} \log _{2} e_{\prec}$, then $|L|=6 \cdot 2^{\frac{1}{2} \log _{2} e_{\prec}-1}+e_{\prec} / \log _{2} e_{\prec}-3 \leq$ $3 \sqrt{e_{\prec}}+e_{\prec} / \log _{2} e_{\prec}$. Thus $|L| / e_{\prec} \rightarrow 0$ for these lattices, showing that an inequality of the form $|L| \geq c e_{\prec}(\mathrm{J}($ Con $\mathbf{L}))$ cannot hold for any $c>0$. Moreover, these parameters also show that

$$
|\mathrm{J}(\mathbf{C o n} \mathbf{L})| \cdot e_{\prec}(\mathrm{J}(\mathbf{C o n} \mathbf{L})) \leq c \cdot|L|^{2}
$$

fails for all $c$.

Finally, if $m$ is even, let $\mathbf{P}$ be the height 1 ordered set with $m / 2$ minimal elements, $m / 2$ maximal elements, and each minimal element below each maximal element. Then $e_{\prec}=m^{2} / 4$ and Corollary 6 shows that if $\mathbf{L}$ is a lattice with $\mathrm{J}(\mathbf{C o n} \mathbf{L}) \cong \mathbf{P}$, then

$$
|L| \geq \frac{m^{2}}{16 \log _{2}(m / 2)}
$$

and it follows that there is no $\alpha$ such that the class of all such $\mathbf{P}$ 's can be represented as $\mathrm{J}(\mathbf{C o n} \mathbf{L})$ with the size of $\mathbf{L}$ in $O\left(m^{2-\alpha}\right)$, as was shown in [9].

\section{Calculating the congruence lattice and an application}

To actually compute Con $\mathbf{L}$ we form the graph $\mathbf{G}(\mathbf{L})$ and use Tarjan's linear time, depth first algorithm [11] to find the $\equiv$-classes and an edge set $E$ satisfying 
$E_{\prec} \subseteq E \subseteq E_{\leq}$. We then find the transitive, reflexive closure, $E_{\leq}$, of $E$. If there are $k$ classes of $\equiv$, we represent congruences as bit vectors of length $k$. Of course the components of such a bit vector correspond to elements of $J($ Con $\mathbf{L})$, since $\mathrm{J}(\mathbf{C o n} \mathbf{L}) \cong \mathbf{G}(\mathbf{L}) / \equiv$. A congruence $\theta$ is represented by the vector which is 1 in those components corresponding to join irreducible congruences below $\theta$. For each $a \in \mathrm{J}(\mathbf{L})$ we calculate the bit vector for $\operatorname{Cg}\left(a, a_{*}\right)$. (This is not just the vector with a 1 only in the component for $\operatorname{Cg}\left(a, a_{*}\right)$; in fact it is here that we need $\left.E_{\leq} \cdot\right)$

This representation is obviously compact. Meet and join are just the bitwise and and $o r$. One can also decide if $x \theta y$ and compute $\mathbf{L} / \theta$ easily. For the latter we find

$$
S_{\theta}=\left\{a \in \mathrm{J}(\mathbf{L}):\left\langle a, a_{*}\right\rangle \notin \theta\right\} .
$$

The join closure of this set is a join subsemilattice of $\mathbf{L}$ which is isomorphic to $\mathbf{L} / \theta$ under the induced order from $\mathbf{L}$. The details are given in [5].

W. Geyer $[6,7]$ conjectured that, if $\mathbf{L}$ is finite and a bounded homomorphic image of a free lattice, then $|L| \leq \mid$ Con $\mathbf{L} \mid$. He tested this conjecture on several examples and noted that when, in addition, $\mathbf{L}$ was subdirectly irreducible, equality held in all his examples. He asked me to use my computer program to try to find a counterexample. Associated with each completely join irreducible element of a free lattice is a finite, subdirectly irreducible lattice which is a bounded homomorphic image of a free lattice. Running the program on a wide selection of such elements produced a subdirectly irreducible lattice which is a bounded image of a free lattice with 53 elements whose congruence lattice had 52 elements. With this example in hand, it was not hard to construct a smaller example. An example with $|L|=12$ and $\mid$ Con $\mathbf{L} \mid=11$ is given in Figure 3 .
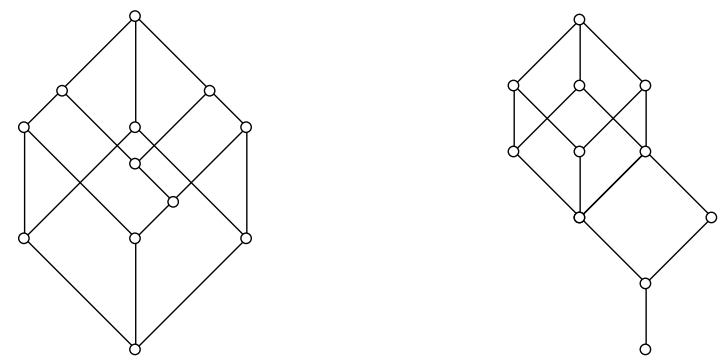

Figure 3. L and Con L.

\section{REFERENCES}

[1] P. Crawley and R. P. Dilworth, Algebraic Theory of Lattices, Prentice-Hall, Englewood Cliffs, New Jersey, 1973.

[2] A. Day, Doubling constructions in lattice theory, Canad. J. Math. 44 (1992), 252-269. MR 93f:06008

[3] R. P. Dilworth, The arithmetical theory of Birkhoff lattices, Duke Math. J. 8 (1941), 286-299. MR 3:100a

[4] R. P. Dilworth and M. Hall, The embedding theorem for modular lattices, Ann. of Math. 45 (1944), 450-456. MR 6:33c

[5] R. Freese, J. Ježek, and J. B. Nation, Free Lattices, Amer. Math. Soc., Providence, 1995, Mathematical Surveys and Monographs, vol. 42. MR 96c:06013

[6] W. Geyer, The class of lattices with $|L|=|\operatorname{Con}(L)|$, General algebra and application, Heldermann, Berlin, 1993, pp. 86-88. MR 94c:06012

[7] W. Geyer, On Tamari lattices, Discrete Math. 133 (1994), 99-122. MR 95m:06021 
[8] G. Grätzer, H. Lakser, and E. T. Schmidt, Congruence lattices of small planar lattices, Proc. Amer. Math. Soc. 123 (1995), 2619-2623. MR 95k:06017a

[9] G. Grätzer, I. Rival, and N. Zaguia, Small representations of finite distributive lattices as congruence lattices, Proc. Amer. Math. Soc. 123 (1995), 1959-1961; Correction, Proc. Amer. Math. Soc., to appear. MR 95k:06017b

[10] R. Medina and L. Nourine, Algorithme efficace de génération des idéaux d'un ensemble ordonné, C. R. Acad. Sci. Paris Sér. I Math. 319 (1994), 1115-1120. MR 95i:06006

[11] R. E. Tarjan, Depth first searches and linear graph algorithms, SIAM J. of Computing 1 (1972), 146-160. MR 46:3313

Department of Mathematics, University of Hawail, Honolulu, Hawail 96822

E-mail address: ralph@math.hawaii.edu 